OPEN ACCESS

Edited by:

André Schmidt,

University of Basel, Switzerland

Reviewed by:

Matthew J. Hoptman, Nathan Kline Institute for Psychiatric

Research, United States

Chun Wang,

Nanjing Brain Hospital Affiliated to

Nanjing Medical University, China

${ }^{*}$ Correspondence:

Yonggui Yuan

yygylh2000@sina.com

Zhenghua Hou

drhouzh@seu.edu.cn

Specialty section

This article was submitted to

Neuroimaging and Stimulation,

a section of the journal

Frontiers in Psychiatry

Received: 02 October 2021 Accepted: 06 December 2021

Published: 18 January 2022

Citation:

Hou Z, Li T, He X, Zhang Y, Chen H, Jiang W, Yin $Y$ and Yuan $Y(2022)$

Distinct Features of Cerebral Blood Flow and Spontaneous Neural Activity as Integrated Predictors of Early

Response to Antidepressants.

Front. Psychiatry 12:788398.

doi: 10.3389/fpsyt.2021.788398

\section{Distinct Features of Cerebral Blood Flow and Spontaneous Neural Activity as Integrated Predictors of Early Response to Antidepressants}

\author{
Zhenghua Hou ${ }^{1 *}$, Tong $\mathrm{Li}^{2,3}$, Xiaofu He ${ }^{2}$, Yuqun Zhang ${ }^{1}$, Huanxin Chen ${ }^{4}$, Wenhao Jiang ${ }^{1}$, \\ Yingying Yin ${ }^{1}$ and Yonggui Yuan ${ }^{1 *}$ \\ ${ }^{1}$ Department of Psychosomatics and Psychiatry, Institute of Psychosomatics, School of Medicine, Affiliated Zhongda \\ Hospital, Southeast University, Nanjing, China, ${ }^{2}$ Department of Psychiatry, The New York State Psychiatric Institute, \\ Columbia University Medical Center, New York, NY, United States, ${ }^{3}$ Department of Information Engineering, Harbin Institute \\ of Technology, Harbin, China, ${ }^{4}$ Key Laboratory of Cognition and Personality, Ministry of Education, School of Psychology, \\ Southwest University, Chongqing, China
}

Aims: The purpose of this study is to explore whether pre-treatment features of brain function can discriminate non-responders to antidepressant medication in the early phase.

Methods: Forty-four treatment-responsive depressed (RD) patients, 36 non-responsive depressed (NRD) patients, and 42 healthy controls (HCs) were recruited. Regional cerebral blood flow (CBF) and amplitude of low-frequency fluctuation (ALFF) values were calculated for all subjects. Correlation analyses were used to explore the relationship between symptom improvement and CBF/ALFF. Receiver operating characteristics $(\mathrm{ROC})$ and the 10-fold cross-validation support vector machine (SVM) classifier were applied for the discrimination of treatment response.

Results: Compared with the HCs, the RD and NRD groups exhibited lower CBF and ALFF in the right posterior lobe of the cerebellum. Compared with the NRD group, the $\mathrm{RD}$ group showed distinct CBF patterns in the left frontal striatal regions and right frontal cerebellar regions, as well as distinct ALFF features in the left frontoparietal striatum and right frontotemporal striatal cerebellar regions. The ROC and SVM classifier revealed the optimal power to distinguish the RD and NRD groups based on the combined measures (i.e., CBF and ALFF).

Conclusion: Distinct features of CBF and ALFF in the frontal striatal network may serve as promising neuroimaging predictors for identifying patients with blunted responsiveness, which may facilitate personalized antidepressant treatment.

Keywords: predictors, early antidepressants response, support vector machine, cerebral blood flow, amplitude of low-frequency fluctuation 


\section{INTRODUCTION}

Despite their importance for treating major depressive disorder (MDD), antidepressants can relieve only $22-50 \%$ of patients, and $<33 \%$ of patients achieve clinical remission $(1,2)$. Identifying patients with early improvement potential before initiating medication can not only minimize unnecessary ineffective treatments but also optimize treatment compliance (3). Convergent evidence shows that early symptom improvement can robustly predict subsequent remission of MDD (4-7), while early non-responsiveness may indicate ultimate nonremission in response to antidepressants $(8,9)$. Therefore, the researchers suggest to advance the time window for evaluating early responses to 2 weeks $(10,11)$. In summary, exploring the prospective indicators of early response could optimize the "trial and error" method in current clinical routines (12).

Advances in resting-state functional magnetic resonance imaging (rs-fMRI) have substantially facilitated the identification of neuropathological features of depression (13-15). Emerging evidence has shown that spontaneous brain activity, which is reflected by the amplitude of low-frequency fluctuation (ALFF), is significantly different between MDD patients and healthy subjects $(13,16,17)$. Compared with the measure of regional homogeneity, which describes the similarity of a given voxel time series to its nearest 26 neighbors (18), the ALFF was more suitable for measuring regional brain activity in the present study. Meanwhile, arterial spin labeling (ASL) imaging can reflect cerebral blood flow (CBF) via the noninvasive marking of arterial blood flow with an endogenous perfusion agent $(19,20)$. This method does not use a contrast tracer and is based upon the subtraction of two consecutively generated images. Accumulating evidence indicates the potential relevance of regional $\mathrm{CBF}$ and spontaneous activity $(21,22)$. However, it remains uncertain whether local hemodynamic changes and regional activities are altered simultaneously in $\operatorname{MDD}(23,24)$.

Furthermore, some studies have suggested that pre-treatment functional features are distinct between responders and nonresponders to antidepressant treatment (25). Meanwhile, several results have reported that antidepressants may impact cerebral perfusion in healthy subjects $(26,27)$, as well as the corticosubcortical perfusion in remitted or treatment-resistant MDD patients $(20,28)$. However, predicting the antidepressant response using altered brain function in the pre-treatment phase has been challenging (29), and there is no study that has combined quantitative information from the $\mathrm{CBF}$ and ALFF to predict early antidepressants response. Therefore, the identification of multimodal predictors of early response may help bridge the gap between baseline imaging features and treatment efficacy (30).

We previously revealed that altered resting-state functions within the reward circuit and the default mode network (DMN) could allow for the identification of patients with unfavorable antidepressant responses $(31,32)$. In this study, we aimed to extend our previous research by predicting early treatment responsiveness [i.e., responsive depression (RD) or nonresponsive depression (NRD)] using discriminative $\mathrm{CBF}$ and
ALFF features and machine learning method. We also explored whether the abnormal CBF and ALFF in emotion-processing regions changed synchronously in MDD. We hypothesized that the alterations of $\mathrm{CBF}$ and ALFF are mainly located in frontal-limbic and subcortical regions, which are closely related to self-referential thought and maladaptive rumination in depression.

\section{MATERIALS AND METHODS}

\section{Participants}

The participants were recruited from the Affiliated Zhongda Hospital, Southeast University, and the local community. The Southeast University Research Ethics Committee approved the research, and an informed consent form was signed by all subjects. All participants received MRI contradiction screening, demographic data, and clinical interviews. A consensus was reached by two trained experienced psychiatrists to diagnose MDD using the Structured Clinical Interview for DSM-IV Axis I Disorders (SCID-I/P), clinician version (33). The inclusion and exclusion criteria are the same as the items in our previous study (31) (more details are listed in the Supplementary Material). All participants are right-handed unequivocally and naturally. The MRI scans were processed before the administration of antidepressant treatment. During the 2-week follow-up period, five patients withdrew from the study. Finally, $80 \mathrm{MDD}$ patients and 42 heathy controls (HCs) completed the procedures (2-week follow-up) and underwent the quality control (e.g., head motion and ghost intensity). According to the Hamilton Depression Scale (HAMD) score reduced by $50 \%$, the MDD subjects were divided into the RD $(n=44)$ and NRD groups $(n=36)$ after 2 weeks of antidepressant treatment.

\section{Brain Image Acquisition}

All participants were applied a 12-channel head coil to perform rs-fMRI and ASL imaging scans on a 3.0 Tesla scanner (Siemens Medical Systems, Erlangen, Germany). The subjects lie on their backs, and their heads are tightly secured with straps and foam pads to minimize potential head movement. All subjects were instructed to close their eyes, relax, and stay awake and not to consider any specific things during the scan. The details of MRI sequences are illustrated in the Supplementary Material.

\section{Functional Image Preprocessing Protocol}

Resting-state functional images were preprocessed utilizing the Data Processing Assistant for Resting-State Function (DPARSF 2.3 Advanced edition) MRI toolkit (34), which combined procedures based on the Resting-State Functional MRI toolkit (REST, http://www.restfmri.net) (35), and statistical parametric mapping software package (SPM8, http://www.fil.ion.ucl.ac.uk/ spm). Briefly, the slice timing, head motion checking, coregistering, spatial normalization, spatial smoothing, detrending, and nuisance signal (white matter, cerebrospinal fluid signal, rigid body-six-corrected head movement parameters) regression were conducted. After the above preprocessing, the averaged square root was obtained across $0.01-0.08 \mathrm{~Hz}$ at each voxel and the ALFF generated at the given voxel. Finally, for 
standardization procedure, ALFF was transformed to $Z$ score by subtracting the global mean value and then divided by the standard deviation (36). The details are elaborated in the Supplementary Material.

The scrubbing was finally performed using the DPARSF. According to a previous study (37), if the volumes with framewise displacement (FD) larger than $0.5 \mathrm{~mm}$ with prior one and later two volumes will be deleted, then subjects with fewer than 4 min of remaining data (about 50\% volumes) will be excluded.

To examine the potential confounder of head motion, we employed the mean FD (38) to control the contamination of head motion in group-level comparison. The FD measures the comparative head motion of each timepoint relative to the prior timepoint, by integrating six framewise head motion parameters (39).

The ASL image data were reconstructed and checked by two senior radiologists. T1 images were manually inspected for quality control, and artifacts were removed before preprocessing. The qualified PASL data was processed using the ASLtbx (40) and SPM12 (http://www.fil.ion.ucl.ac.uk/spm). The ALFF maps of each subject were transformed to $z$-maps with the REST software (http://www.restfmri.net) for further analysis. The results were corrected with the $3 \mathrm{dCl}$ (ustSim program. For details, see Supplementary Material.

\section{Statistical Analyses}

Continuous variables are presented as the mean \pm standard deviation (SD). The analysis of covariance (ANCOVA), posthoc test, independent two-sample $t$-test, and chi-square test (Statistical Package for the Social Sciences software, SPSS19.0, Chicago) were used to confirm significant differences in demographic data and HAMD scores among the RD, NRD, and $\mathrm{HC}$ groups. The imaging calculations were conducted in REST (35), and the results were corrected with the 3dClustSim program (https://afni.nimh.nih.gov/pub/dist/doc/program_ help/ 3dClustSim.html). The group imaging comparison, correlation analyses, receiver operating characteristic (ROC), and support vector machine (SVM) analyses are detailed in the Supplementary Material. In this study, the "fingerprint" was employed to display three or more quantitative variable data in the form of a two-dimensional graph, which can visually illustrate the group data on an axis starting from the same point. Unless otherwise specified, the threshold of statistical significance was set as $P<0.05$.

\section{RESULTS}

\section{Sample Characteristics}

No significant differences in age, sex, or education levels were detected among the RD, NRD, and HC groups (all $P>0.05$, Table 1), and no significant differences in baseline severity of depression (i.e., HAMD scores) were revealed between the RD and NRD groups $(t=-0.477, P=0.635)$. Furthermore, no significant differences in mean FD were exhibited among the three groups $(P=0.989)$.

\section{The Common and Distinct Features of Regional CBF and ALFF Among the RD and NRD Groups}

As detailed in Figure 1, both the RD and NRD groups exhibited lower CBF in the left lingual gyrus and right posterior lobe of the cerebellum compared with the HC group $(P<0.05$, 3dClustSim correction). The RD and NRD groups also showed lower ALFF in the bilateral sensorimotor cortex, bilateral middle occipital gyrus (MOG), and right posterior lobe of the cerebellum but presented higher ALFF in the bilateral middle frontal gyri (MFG) and lentiform nucleus when compared with the HC group $(P<$ 0.05, 3dClustSim correction).

Distinct patterns of regional CBF and ALFF were also observed in the RD and NRD groups when compared with the HC group (Figure 1; Supplementary Tables S1, S2). Specifically, the $\mathrm{RD}$ group showed distinctly lower ALFF levels in the left postcentral gyrus (L_PoCG) and superior temporal gyrus (R_STG) but enhanced ALFF levels in the left precentral gyrus (L_PreCG) and left parahippocampal gyrus relative to the $\mathrm{HC}$ group.

In contrast to the $\mathrm{RD}$ group, the $\mathrm{NRD}$ group showed significantly lower CBF levels in the left superior frontal gyrus (L_SFG), right calcarine gyrus, and left cerebellum posterior lobe (L_CePL) but higher $\mathrm{CBF}$ in the right middle temporal gyrus (R_MTG), right middle/superior frontal gyrus, and R_PoCG. As for the rs-fMRI modality, the NRD group exhibited lower ALFF levels in the right calcarine gyrus and right precuneus but greater

TABLE 1 | Demographic and clinical characteristics of all participants (mean \pm SD).

\begin{tabular}{|c|c|c|c|c|c|c|}
\hline Group & $\mathrm{RD}(n=44)$ & NRD $(n=36)$ & $\mathrm{HC}(n=42)$ & Statistic value & $P$ value & Effect size \\
\hline Education (years) & $9.27 \pm 4.00$ & $9.33 \pm 4.28$ & $11.02 \pm 3.79$ & $F_{(2,119)}=2.008$ & $0.139^{a}$ & $f=0.20$ \\
\hline Age (years) & $48.75 \pm 13.31$ & $47.64 \pm 15.75$ & $47.94 \pm 17.62$ & $F_{(2,119)}=0.112$ & $0.894^{a}$ & $f=0.03$ \\
\hline Gender (F/M) & $32 / 12$ & $27 / 9$ & $23 / 19$ & $\chi^{2}=4.515, \mathrm{df}=2$ & $0.105^{b}$ & $w=0.19$ \\
\hline HAMD & $28.93 \pm 7.35$ & $29.67 \pm 6.18$ & NA & $t=-0.477, \mathrm{df}=78$ & 0.635 & $d=0.11$ \\
\hline Handedness & Right & Right & Right & NA & NA & NA \\
\hline Mean FD & $0.107 \pm 0.034$ & $0.108 \pm 0.029$ & $0.108 \pm 0.043$ & $F_{(2,119)}=0.011$ & 0.989 & NA \\
\hline
\end{tabular}

For comparison of demographics: a $P$ values were obtained using one-way ANOVA tests; ${ }^{b} P$ value for the gender distribution among three groups was obtained using chi-square test. $P<0.05$ was considered significant. HC, healthy controls; NRD, non-responsive depression; RD, responsive depression; HAMD, Hamilton Depression Rating Scale; F/M, female/male; $N A$, not applicable; FD, framewise displacements. Parametric values are represented as the mean $\pm S D$ (standard deviation). 


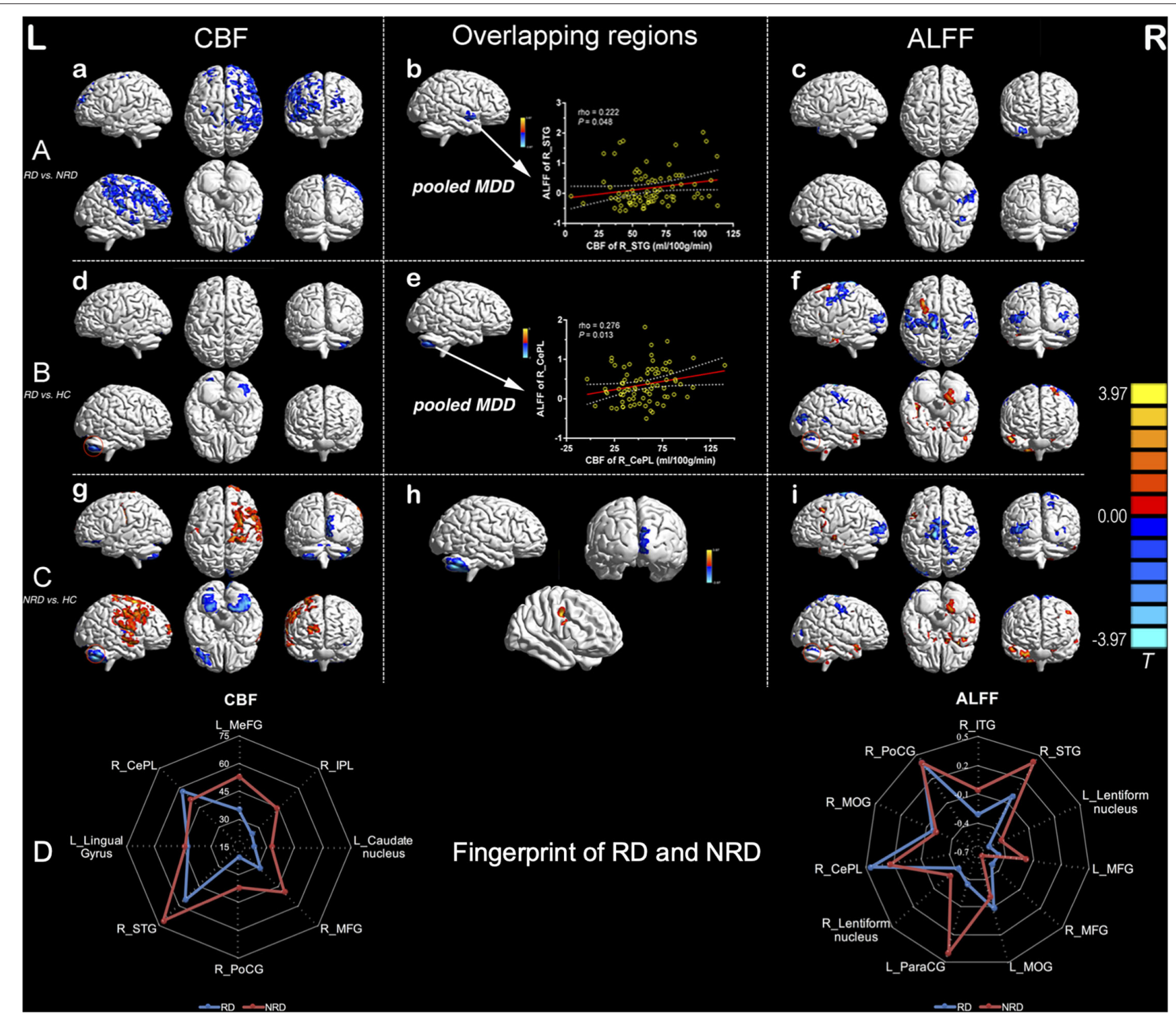

FIGURE 1 | Inter-group regional differences in CBF and ALFF and positive correlations between CBF and ALFF values of overlapping regions (R_STG and R_CePL) in the pooled depression group. Row A: differences in regional CBF (a) and ALFF (c) between the RD and NRD and overlapping regions with significant differences between the CBF and ALFF modalities in pooled MDD (b). Row B: differences in regional CBF (d) and ALFF (f) between RD and HC and overlapping regional alterations in CBF and ALFF in pooled MDD (e). Row C: regional differences in CBF (g) and ALFF (i) between the NRD and HC and overlapping regions of the CBF and ALFF measures (h); row D: the distinctive fingerprints of regional CBF and ALFF between the RD and NRD groups. The red circles in (d) and (g) indicate lower CBF and ALFF in R_CePL in both the RD and NRD groups, as compared with the HC group. The threshold was set at a corrected $P<0.05$, and the $T$-score bar is present on the right side. CBF, cerebral blood flow; ALFF, amplitude of low-frequency fluctuation; L, left; R, right; HC, healthy controls; NRD, non-responsive depression; RD, responsive depression; STG, superior temporal gyrus; CePL, cerebellum posterior lobe.

ALFF levels in the left inferior frontal gyrus (L_IFG), R_PreCG, and R_STG compared with the HC group.

\section{The Distinct Fingerprints of Regional CBF and ALFF Between the RD and NRD Groups}

To visually and comprehensively show the different patterns of $\mathrm{CBF}$ and ALFF between the RD and NRD groups, fingerprints were used to illustrate the regional changes in the ASL and BOLD modalities. Compared with the NRD group, the RD group showed distinct CBF patterns in the left frontal striatal area and right frontal cerebellar region and exhibited differential ALFF patterns in the left frontoparietal striatal area and right frontotemporal-striatal-cerebellar regions (Figure 1).

\section{Analysis of the Association Between the Imaging Measures and HAMD Scores}

In the $\mathrm{RD}$ group, the lower ALFF in the right middle occipital gyrus (R_MOG) was negatively related to symptom improvement after 2 weeks of treatment (i.e., the HAMD 
reduction rate) (rho $=-0.362, P=0.016$ ) and positively correlated with the HAMD score in week $2(r=0.335, P=0.026)$ (Figure 2A). The lower ALFF in the L_MOG was negatively related to symptom improvements (rho $=-0.310, P=0.040$ ) (Figure 2B). The higher ALFF of the L_lentiform nucleus was negatively related to the HAMD score in week 2 (rho $=-0.363$, $P=0.016$ ) but positively related to symptom improvement (rho $=0.360, P=0.016$ ) (Figure 2C).

Additionally, in the NRD group, the baseline HAMD score was positively associated with the higher ALFF of the R_STG ( $r=0.343, P=0.040$ ) but negatively correlated with the lower ALFF of the R_CePL $(r=-0.340, P=0.042)$ and the CBF of the R_calcarine gyrus (rho $=-0.363, P=0.030$ ) (Figures 2D,E), respectively. A significant negative correlation was also found between the CBF of the L_caudate nucleus and symptom improvement (rho $=-0.358, P=0.032)$ (Figure $2 \mathrm{~F}$ ). Specifically, in the pooled MDD group (which included both the $\mathrm{RD}$ and NRD patients), the ALFF of the R_inferior temporal gyrus (R_ITG) was positively related to the HAMD score at week 6 (rho $=0.372, P<0.001$ ) but negatively correlated with the reduction of HAMD score (rho $=-0.389, P<0.001$ ) (Figure 2G).

\section{The Predictive Performance of Regional CBF and ALFF Changes in Distinguishing the NRD From RD}

The ROC analyses revealed that the areas under the curve (AUCs) of the combined measures in each modality (i.e., $\mathrm{CBF}$ and ALFF) were 0.749 and 0.757 , respectively (Figures 3A,B). When

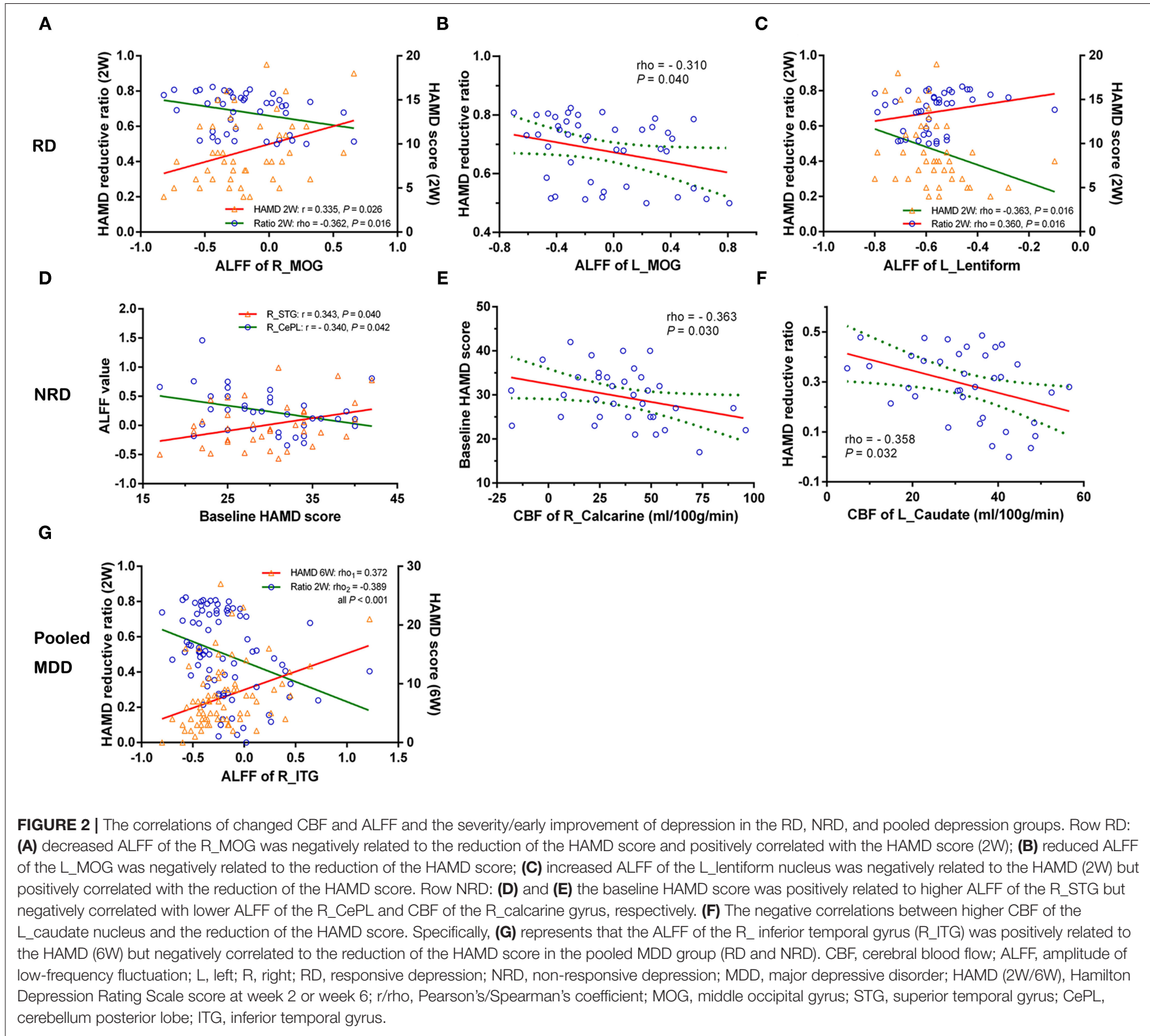


A

ASL

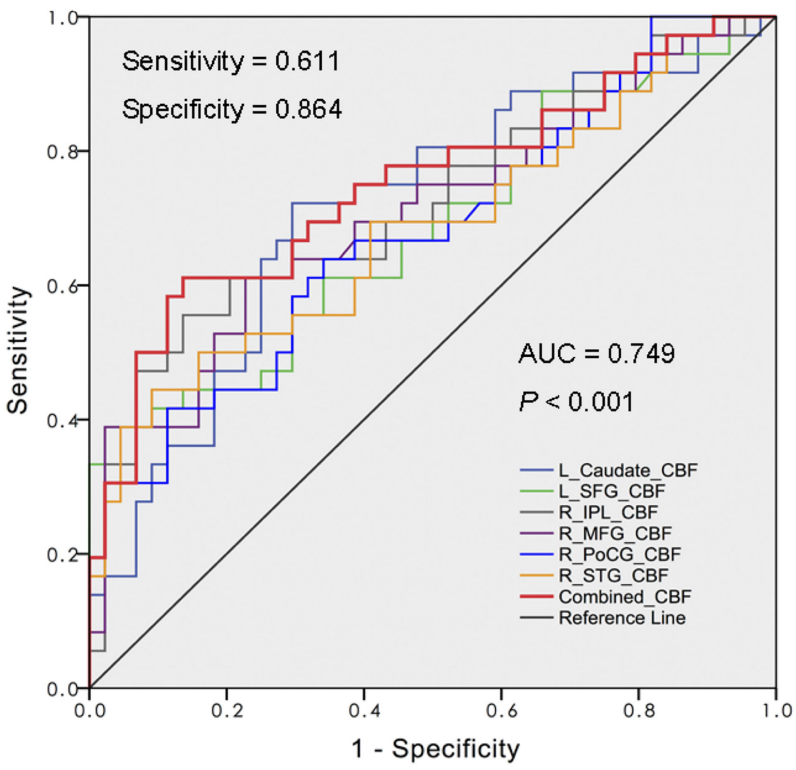

C

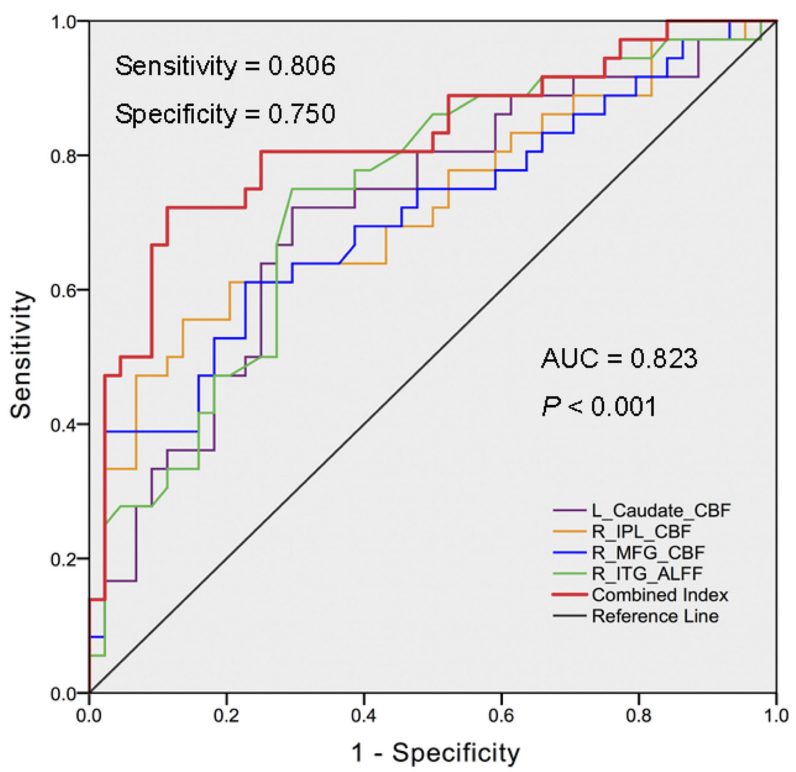

B

ALFF

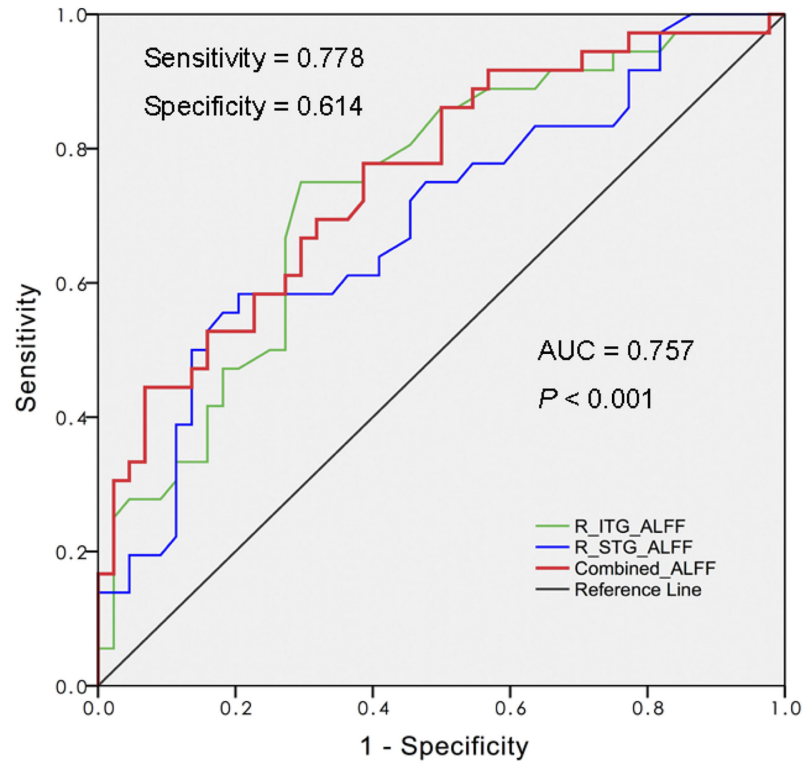

SVM

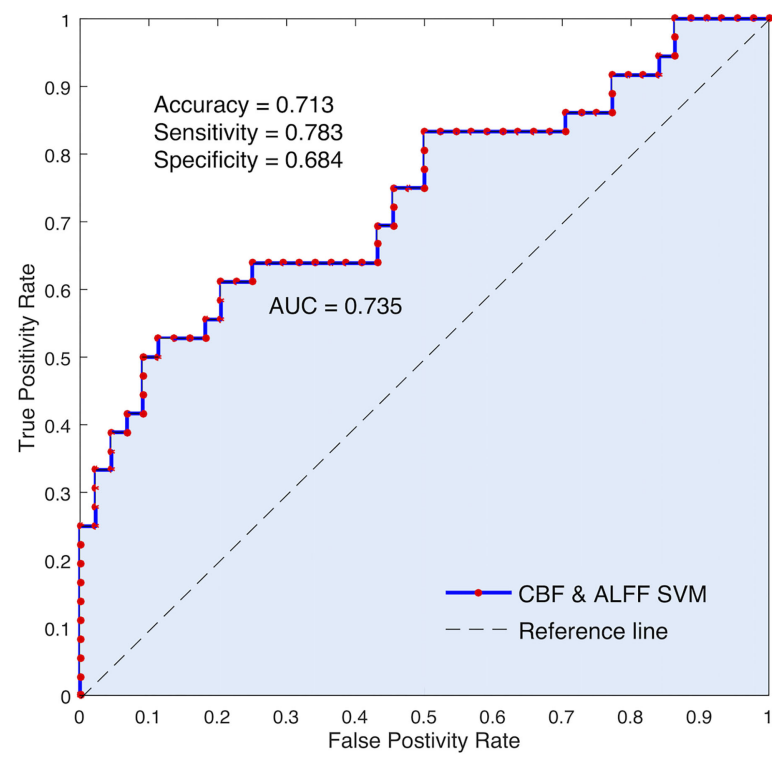

FIGURE 3 | Differentiation analyses using regional CBF, ALFF, and integrated bi-modal parameters. (A) The performance of changed CBF values in differentiating the NRD from RD; (B) the performance of altered ALFF values in segregating the NRD from RD; (C) the optimal performance of the combination of changed CBF and ALFF values in distinguishing the NRD from RD; (D) the accuracy of the combined eight features (the CBF in L_caudate nucleus, R_SFG, R_IPL, R-MFG, R_STG, and R_PoCG, as well as the ALFF in R_STG and R_ITG) using 10-fold cross-validation SVM classifier. ROC, receiver operator characteristic; NRD, non-responsive depression; RD, responsive depression; AUC, area under the curve; CBF, cerebral blood flow; ALFF, amplitude of low-frequency fluctuation; L, left; R, right; SFG, superior frontal gyrus; IPL, inferior parietal lobule; MFG, middle frontal gyrus; PoCG, postcentral gyrus; STG, superior temporal gyrus; ITG, inferior temporal gyrus; SVM, support vector machine.

the measures of the two modalities (ASL and rs-fMRI) were integrated, they showed optimal performance for discriminating the NRD group from the RD group (AUC $=0.823, P<$
$0.001)$, with balanced specificity (89\%) and sensitivity (72\%) (Figure 3C). The ROC results of each regional measure are listed in Supplementary Table S3. 
We further confirmed the classification power of the above eight features (CBF in the L_caudate nucleus, R_IPL, R_SFG, RMFG, R_STG, and R_PoCG and ALFF in the R_ITG and R_STG) using a 10-fold cross-validation SVM classifier. The results revealed that the combination of eight features can discriminate the NRD patients with a moderate accuracy of 0.713 (AUC = 0.735 , sensitivity $=0.783$, specificity $=0.684)($ Figure 3D).

\section{DISCUSSION}

In the present study, we primarily detected notable differences in widespread regions between the NRD and RD groups in the rsfMRI and ASL modalities. Specifically, the fingerprint differences between the RD and NRD patients are mainly characterized by the CBF patterns in the left frontal striatal and right frontal cerebellar areas and by the ALFF features in the left frontoparietal striatal regions and the right frontotemporal striatal-cerebellar areas, respectively. The regional changes in CBF or ALFF in several regions were significantly correlated with depression severity or symptom improvement. Importantly, the ROC and SVM analyses using bi-modal (i.e., rs-fMRI and ASL) features further confirmed the optimal performance in differentiating NRD subjects, indicating the potential value of integrated imaging markers in predicting early antidepressant responses.

In the pooled MDD, the overlapping changes in the $\mathrm{CBF}$ and ALFF in the cerebellum when compared with the $\mathrm{HC}$, which indicate that there were concomitant abnormalities in both blood perfusion and spontaneous activity in depression and further support the opinion that regional CBF is closely related to the intrinsic activity during emotion processing $(22,41)$. The cerebellum reciprocally connects with the limbic regions (42) and the DMN (43), which are responsible for emotion regulation and reappraisal (44). Imaging studies have substantiated that abnormal volume, brain activity, and interhemispheric coordination of the cerebellum are implicated in the pathophysiology and treatment of MDD $(45,46)$. Our results revealed that the lower ALFF in R_CePL was negatively related to the baseline severity of depression, further indicating that cerebellar dysfunction may be the neuropathological basis that affects the severity of depression.

Additionally, the present study found consistently lower CBF and ALFF in the occipital regions (left lingual gyrus, right calcarine gyrus, and bilateral MOG) and the sensorimotor regions in both the $\mathrm{RD}$ and $\mathrm{NRD}$ groups compared with the HC group. Those regions are considered essential components of bottom-up selective attention and visual perception in emotion regulation $(47,48)$. Aberrant activities in visual recognition circuits are deeply implicated in the dysregulation of facial emotion processing and pessimistic social interaction, mediating core symptoms of depression such as anhedonia and social oversensitivity $(49,50)$. Moreover, previous studies also confirmed reduced ALFF of the lingual gyrus and MOG in non-responsive (17) and treatment-resistant subjects (51). The present results extend the prior viewpoint that the dysfunction of facial emotion perception might partly constitute the neural substrates of MDD, which suggests a potential target for optimized medication.
Importantly, as an integral component of the DMN underlying the pathological rumination in depression, the R_STG showed lower CBF and ALFF in the RD group compared with the NRD group but did not differ significantly from the HC group. Evidence from functional imaging studies suggested that a higher CBF in the STG was critical to maintaining static DMN function in healthy subjects $(52,53)$. Furthermore, some fMRI studies have also found that the ALFF in the STG was reduced in treatment-naive MDD (14) but was increased in the severe subtype of MDD with suicidality (54). Considering that an overactivated DMN substantially mediates maladaptive rumination and preferential attention bias to negative information in MDD (55), it would be reasonable to speculate that an abnormal hyperactivity of the STG is an essential neural substrate of severe depressive symptoms (e.g., intense suicidality) and inadequate treatment efficacy. Patients with favorable antidepressant efficacy may recruit compensatory downregulation of the STG to facilitate a positive reaction to antidepressant treatment (56).

The fingerprint patterns also revealed distinct CBF/ALFF changes between the RD and NRD groups, indicative of the involvement of spatially orchestrated neural ensembles in the neural mechanism of MDD. Those widespread regions are considered crucial mediators that can synergistically modulate the top-down process of emotion regulation and antidepressant efficacy in $\operatorname{MDD}(55,57,58)$. Importantly, the combined ROC analysis and 10-fold cross-validation SVM classification were further conducted. The results revealed stable performance with an optimal effect in discriminating the NRD from the $\mathrm{RD}$ group. In line with the evidence mentioned above $(3,58)$, the results further substantiated that multiple regions, rather than a specific single region, can mediate emotion regulation and identify patients with distinct neural features that may respond differently to antidepressant therapy.

As a critical nucleus of the corticostriatal circuit that is profoundly linked to emotion regulation, the caudate shows microstructural and functional aberrations in depressed patients (59) and in rats exposed to chronic mild stress (60). Furthermore, a recent study reported that reduced resting-state activity in the caudate could predict clinical remission in MDD after a single dose of antidepressants (61), suggesting that overactivation of the caudate could be selected as a potential target for antidepressants. Interestingly, the higher $\mathrm{CBF}$ of the L_caudate nucleus was also inversely correlated with symptom improvement in the NRD group. Our results further validated the prominent role of the caudate nucleus in antidepressant efficacy from the dimension of quantitative CBF. We also found that the ALFF of the R_ITG was positively related to the severity of depression in week 6 but was negatively correlated with early symptom improvement. The ITG is considered a pivotal region in the neuropathology of MDD, and recent rs-fMRI studies have detected abnormal network heterogeneity (62) and brain activation (63) of the R_ITG in MDD and dysfunction of the ITG in subthreshold depression (64). It is noteworthy that the ALFF value of the R-ITG in the $\mathrm{RD}$ group was significantly lower than that in the NRD group, indicating that the downregulation of spontaneous activity in the R-ITG might facilitate the improvement of depressive symptoms. 
Some potential limitations should be considered when evaluating these results appropriately. First, the present study was designed without an MRI scan at the end of week 2, so whether these regional CBF/ALFF changes persist or could be reversed by antidepressant treatment needs to be further confirmed. Future studies using a longitudinal design are required to verify whether the group differences in the brain are causal or casual. Second, different antidepressants were prescribed in this study, so the prediction of treatment response to a specific antidepressant cannot be confirmed. Further studies investigating the predictors of early treatment response would benefit from the prescription of homogeneous types of antidepressants (e.g., SSRIs or SNRIs).

\section{CONCLUSION}

In summary, the present study suggests that dysfunctions in distributed regions are orchestrated in the neuropathological processes of $\mathrm{MDD}$, and the regional alterations of $\mathrm{CBF}$ and ALFF in the frontal striatal area may be promising markers for the prediction of short-term treatment outcomes. Meanwhile, the distinct alteration of the posterior lobe of the cerebellum [functionally connected to the DMN (65)] in the pooled MDD group may be a common trait in depression that can be considered a potential diagnostic marker of MDD. Although these findings need further validation before clinical application, they provide theoretical evidence for research domain criteria (RDoC) that adopt quantitative imaging features to predict efficacy during the early stage.

\section{DATA AVAILABILITY STATEMENT}

The raw data supporting the conclusions of this article will be made available by the authors, without undue reservation.

\section{ETHICS STATEMENT}

The studies involving human participants were reviewed and approved by Southeast University Research

\section{REFERENCES}

1. Lu J, Xu X, Huang Y, Li T, Ma C, Xu G, et al. Prevalence of depressive disorders and treatment in China: a cross-sectional epidemiological study. Lancet Psychiatry. (2021) 8:981-90. doi: 10.1016/S2215-0366(21) 00251-0

2. Scangos KW, Khambhati AN, Daly PM, Makhoul GS, Sugrue LP, Zamanian H, et al. Closed-loop neuromodulation in an individual with treatment-resistant depression. Nat Med. (2021) 27:1696-700. doi: 10.1038/s41591-021-01480-w

3. Spies M, Kraus C, Geissberger N, Auer B, Klobl M, Tik M, et al. Default mode network deactivation during emotion processing predicts early antidepressant response. Transl Psychiatry. (2017) 7:e1008. doi: 10.1038/tp.2016.265

4. Vermeiden M, Kamperman AM, Vulink ME, van den Broek WW, Birkenhager TK. Early improvement as a predictor of eventual antidepressant treatment response in severely depressed inpatients. Psychopharmacology. (2015) 232:1347-56. doi: 10.1007/s00213-014-3765-1

5. Sakurai H, Uchida H, Abe T, Nakajima S, Suzuki T, Pollock BG, et al. Trajectories of individual symptoms in remitters versus non-remitters with depression. J Affect Disord. (2013) 151:506-13. doi: 10.1016/j.jad.2013.06.035
Ethics Committee. The patients/participants provided their written informed consent to participate in this study.

\section{AUTHOR CONTRIBUTIONS}

$\mathrm{YYu}$ and $\mathrm{ZH}$ designed the study, had full access to the integrated data in the study, and took responsibility for the analysis of the data. WJ and YYi collected the clinical and MRI data. ZH and YZ performed the literature search, imaging data analyses, statistical analysis, and wrote the first draft of the manuscript. TL and $\mathrm{XH}$ helped to perform the machine learning analysis. All authors contributed to the article and approved the submitted version.

\section{FUNDING}

This study was supported by Natural Science Foundation of Jiangsu Province (No. BK20201270 to ZH), National Natural Science Foundation of China (No. 81971277 to YYu and No.81801349 to YYi), Jiangsu Provincial Key Research and Development Program (No. BE2019748 to YYu), and State Scholarship Fund of China Scholarship Council (No. 201706090193 to $\mathrm{ZH})$.

\section{ACKNOWLEDGMENTS}

The authors appreciate all participants for their cooperation in this study. The authors also gratefully acknowledge the work of the MR Center, Affiliated Zhongda Hospital of Southeast University.

\section{SUPPLEMENTARY MATERIAL}

The Supplementary Material for this article can be found online at: https://www.frontiersin.org/articles/10.3389/fpsyt. 2021.788398/full\#supplementary-material

6. Gorwood P, Demyttenare K, Vaiva G, Corruble E, Llorca PM, Bayle F, et al. An increase in joy after two weeks is more specific of later antidepressant response than a decrease in sadness. J Affect Disord. (2015) 185:97103. doi: 10.1016/j.jad.2015.06.019

7. Szegedi A, Muller MJ, Anghelescu I, Klawe C, Kohnen R, Benkert O. Early improvement under mirtazapine and paroxetine predicts later stable response and remission with high sensitivity in patients with major depression. J Clin Psychiatry. (2003) 64:413-20. doi: 10.4088/JCP.v64n0410

8. Nierenberg AA, McLean NE, Alpert JE, Worthington JJ, Rosenbaum JF, Fava M. Early nonresponse to fluoxetine as a predictor of poor 8-week outcome. Am J Psychiatry. (1995) 152:1500-3. doi: 10.1176/ajp.152.10.1500

9. Grieve SM, Korgaonkar MS, Gordon E, Williams LM, Rush AJ. Prediction of nonremission to antidepressant therapy using diffusion tensor imaging. J Clin Psychiatry. (2016) 77:e436-43. doi: 10.4088/JCP.14m09577

10. Kudlow PA, McIntyre RS, Lam RW. Early switching strategies in antidepressant non-responders: current evidence and future research directions. CNS Drugs. (2014) 28:601-9. doi: 10.1007/s40263-014-0171-5

11. Szegedi A, Jansen WT, van Willigenburg AP, van der Meulen E, Stassen HH, Thase ME. Early improvement in the first 2 weeks as a predictor of treatment 
outcome in patients with major depressive disorder: a meta-analysis including 6562 patients. J Clin Psychiatry. (2009) 70:344-53. doi: 10.4088/JCP.07m03780

12. Kennis M, Gerritsen L, van Dalen M, Williams A, Cuijpers P, Bockting C. Prospective biomarkers of major depressive disorder: a systematic review and meta-analysis. Mol Psychiatry. (2020) 25:321-38. doi: 10.1038/s41380-019-0585-Z

13. Liu F, Guo W, Liu L, Long Z, Ma C, Xue Z, et al. Abnormal amplitude low-frequency oscillations in medication-naive, first-episode patients with major depressive disorder: a resting-state fMRI study. J Affect Disord. (2013) 146:401-6. doi: 10.1016/j.jad.2012.10.001

14. Liu J, Ren L, Womer FY, Wang J, Fan G, Jiang W, et al. Alterations in amplitude of low frequency fluctuation in treatment-naive major depressive disorder measured with resting-state fMRI. Hum Brain Mapp. (2014) 35:4979-88. doi: 10.1002/hbm.22526

15. Orosz A, Jann K, Federspiel A, Horn H, Hofle O, Dierks T, et al. Reduced cerebral blood flow within the default-mode network and within total gray matter in major depression. Brain Connect. (2012) 2:30310. doi: 10.1089/brain.2012.0101

16. Zang YF, He Y, Zhu CZ, Cao QJ, Sui MQ, Liang M, et al. Altered baseline brain activity in children with ADHD revealed by resting-state functional MRI. Brain Dev. (2007) 29:83-91. doi: 10.1016/j.braindev.2006.07.002

17. Wang LJ, Kuang WH, Xu JJ, Lei D, Yang YC. Resting-state brain activation correlates with short-time antidepressant treatment outcome in drug-naive patients with major depressive disorder. J Int Med Res. (2014) 42:96675. doi: 10.1177/0300060514533524

18. Zang Y, Jiang T, Lu Y, He Y, Tian L. Regional homogeneity approach to fMRI data analysis. Neuroimage. (2004) 22:394400. doi: $10.1016 /$ j.neuroimage.2003.12.030

19. Detre JA, Wang J, Wang Z, Rao H. Arterial spin-labeled perfusion MRI in basic and clinical neuroscience. Curr Opin Neurol. (2009) 22:34855. doi: 10.1097/WCO.0b013e32832d9505

20. Duhameau B, Ferre JC, Jannin P, Gauvrit JY, Verin M, Millet B, et al. Chronic and treatment-resistant depression: a study using arterial spin labeling perfusion MRI at 3Tesla. Psychiatry Res. (2010) 182:1116. doi: 10.1016/j.pscychresns.2010.01.009

21. Zou Q, Miao X, Liu D, Wang DJ, Zhuo Y, Gao JH. Reliability comparison of spontaneous brain activities between BOLD and CBF contrasts in eyes-open and eyes-closed resting states. Neuroimage. (2015) 121:91105. doi: 10.1016/j.neuroimage.2015.07.044

22. Li Z, Zhu Y, Childress AR, Detre JA, Wang Z. Relations between BOLD fMRIderived resting brain activity and cerebral blood flow. PLOS ONE. (2012) 7:e44556. doi: 10.1371/journal.pone.0044556

23. Jukovskaya N, Tiret P, Lecoq J, Charpak S. What does local functional hyperemia tell about local neuronal activation? J Neurosci. (2011) 31:157982. doi: 10.1523/JNEUROSCI.3146-10.2011

24. O'Herron P, Chhatbar PY, Levy M, Shen Z, Schramm AE, Lu Z, et al. Neural correlates of single-vessel haemodynamic responses in vivo. Nature. (2016) 534:378-82. doi: 10.1038/nature17965

25. Rentzsch J, Adli M, Wiethoff K, Gomez-Carrillo de Castro A, Gallinat J. Pretreatment anterior cingulate activity predicts antidepressant treatment response in major depressive episodes. Eur Arch Psychiatry Clin Neurosci. (2014) 264:213-23. doi: 10.1007/s00406-013-0424-1

26. Chen Y, Wan HI, O'Reardon JP, Wang DJ, Wang Z, Korczykowski M, et al. Quantification of cerebral blood flow as biomarker of drug effect: arterial spin labeling phMRI after a single dose of oral citalopram. Clin Pharmacol Ther. (2011) 89:251-8. doi: 10.1038/clpt.2010.296

27. Klomp A, Caan MW, Denys D, Nederveen AJ, Reneman L. Feasibility of ASL-based phMRI with a single dose of oral citalopram for repeated assessment of serotonin function. Neuroimage. (2012) 63:1695-700. doi: 10.1016/j.neuroimage.2012.07.038

28. Colloby SJ, Firbank MJ, He J, Thomas AJ, Vasudev A, Parry SW, et al. Regional cerebral blood flow in late-life depression: arterial spin labelling magnetic resonance study. Br J Psychiatry. (2012) 200:1505. doi: 10.1192/bjp.bp.111.092387

29. Osuch E, Gao S, Wammes M, Theberge J, Willimason P, Neufeld RJ, et al. Complexity in mood disorder diagnosis: fMRI connectivity networks predicted medication-class of response in complex patients. Acta Psychiatr Scand. (2018) 138:472-82. doi: 10.1111/acps.12945
30. Lener MS, Iosifescu DV. In pursuit of neuroimaging biomarkers to guide treatment selection in major depressive disorder: a review of the literature. Ann N Y Acad Sci. (2015) 1344:50-65. doi: 10.1111/nyas. 12759

31. Hou Z, Kong Y, He X, Yin Y, Zhang Y, Yuan Y. Increased temporal variability of striatum region facilitating the early antidepressant response in patients with major depressive disorder. Prog Neuropsychopharmacol Biol Psychiatry. (2018) 85:39-45. doi: 10.1016/j.pnpbp.2018. 03.026

32. Hou Z, Gong L, Zhi M, Yin Y, Zhang Y, Xie C, et al. Distinctive pretreatment features of bilateral nucleus accumbens networks predict early response to antidepressants in major depressive disorder. Brain Imaging Behav. (2018) 12:1042-52. doi: 10.1007/s11682-017-9773-0

33. First MB, Spitzer RL, Gibbon M, Williams JBW. Structured Clinical Interview for DSM-IV Axis I Disorders (SCID-I). Washington, DC: American Psychiatric Association (1997).

34. Chao-Gan Y, Yu-Feng Z. DPARSF A MATLAB toolbox for "Pipeline" data analysis of resting-state fMRI. Front Syst Neurosci. (2010) 4:13. doi: $10.3389 /$ fnsys.2010.00013

35. Song XW, Dong ZY, Long XY, Li SF, Zuo XN, Zhu CZ, et al. REST: a toolkit for resting-state functional magnetic resonance imaging data processing. PLoS ONE. (2011) 6:e25031. doi: 10.1371/journal.pone.0025031

36. Zou QH, Zhu CZ, Yang Y, Zuo XN, Long XY, Cao QJ, et al. An improved approach to detection of amplitude of low-frequency fluctuation (ALFF) for resting-state fMRI: fractional ALFF. J Neurosci Methods. (2008) 172:13741. doi: 10.1016/j.jneumeth.2008.04.012

37. Ye Q, Chen X, Qin R, Huang L, Yang D, Liu R, et al. Enhanced regional homogeneity and functional connectivity in subjects with white matter hyperintensities and cognitive impairment. Front Neurosci. (2019) 13:695. doi: 10.3389/fnins.2019.00695

38. Power JD, Barnes KA, Snyder AZ, Schlaggar BL, Petersen SE. Spurious but systematic correlations in functional connectivity MRI networks arise from subject motion. Neuroimage. (2012) 59:2142-54. doi: 10.1016/j.neuroimage.2011.10.018

39. Power JD, Mitra A, Laumann TO, Snyder AZ, Schlaggar BL, Petersen SE. Methods to detect, characterize, and remove motion artifact in resting state fMRI. Neuroimage. (2014) 84:320-41. doi: 10.1016/j.neuroimage.2013.08.048

40. Wang Z, Aguirre GK, Rao H, Wang J, Fernandez-Seara MA, Childress $\mathrm{AR}$, et al. Empirical optimization of ASL data analysis using an ASL data processing toolbox: ASLtbx. Magn Reson Imaging. (2008) 26:2619. doi: 10.1016/j.mri.2007.07.003

41. Petzold GC, Albeanu DF, Sato TF, Murthy VN. Coupling of neural activity to blood flow in olfactory glomeruli is mediated by astrocytic pathways. Neuron. (2008) 58:897-910. doi: 10.1016/j.neuron.2008.04.029

42. Phillips JR, Hewedi DH, Eissa AM, Moustafa AA. The cerebellum and psychiatric disorders. Front Public Health. (2015) 3:66. doi: 10.3389/fpubh.2015.00066

43. Pagen LHG, van de Ven VG, Gronenschild E, Priovoulos N, Verhey FRJ, Jacobs HIL. Contributions of cerebro-cerebellar default mode connectivity patterns to memory performance in mild cognitive impairment. J Alzheimers Dis. (2020) 75:633-47. doi: 10.3233/JAD-191127

44. Balsters JH, Laird AR, Fox PT, Eickhoff SB. Bridging the gap between functional and anatomical features of cortico-cerebellar circuits using meta-analytic connectivity modeling. Hum Brain Mapp. (2014) 35:315269. doi: 10.1002/hbm.22392

45. Du L, Wang J, Meng B, Yong N, Yang X, Huang Q, et al. Early life stress affects limited regional brain activity in depression. Sci Rep. (2016) 6:25338. doi: 10.1038/srep25338

46. Depping MS, Schmitgen MM, Bach C, Listunova L, Kienzle J, Kubera $\mathrm{KM}$, et al. Abnormal cerebellar volume in patients with remitted major depression with persistent cognitive deficits. Cerebellum. (2020) 19:76270. doi: 10.1007/s12311-020-01157-z

47. Pourtois G, Schettino A, Vuilleumier P. Brain mechanisms for emotional influences on perception and attention: what is magic and what is not. Biol Psychol. (2013) 92:492-512. doi: 10.1016/j.biopsycho.2012.02.007

48. Fitzgerald PJ. Gray colored glasses: is major depression partially a sensory perceptual disorder? I Affect Disord. (2013) 151:41822. doi: $10.1016 /$ j.jad.2013.06.045 
49. Bubl E, Kern E, Ebert D, Bach M, Tebartz van Elst L. Seeing gray when feeling blue? Depression can be measured in the eye of the diseased. Biol Psychiatry. (2010) 68:205-8. doi: 10.1016/j.biopsych.2010.02.009

50. Fu CH, Williams SC, Brammer MJ, Suckling J, Kim J, Cleare AJ, et al. Neural responses to happy facial expressions in major depression following antidepressant treatment. Am J Psychiatry. (2007) 164:599607. doi: 10.1176/ajp.2007.164.4.599

51. Guo WB, Liu F, Xue ZM, Xu XJ, Wu RR, Ma CQ, et al. Alterations of the amplitude of low-frequency fluctuations in treatment-resistant and treatment-response depression: a restingstate fMRI study. Prog Neuropsychopharmacol Biol Psychiatry. (2012) 37:153-60. doi: 10.1016/j.pnpbp.2012.01.011

52. Zou Q, Wu CW, Stein EA, Zang Y, Yang Y. Static and dynamic characteristics of cerebral blood flow during the resting state. Neuroimage. (2009) 48:51524. doi: 10.1016/j.neuroimage.2009.07.006

53. Raichle ME, MacLeod AM, Snyder AZ, Powers WJ, Gusnard DA, Shulman GL, et al. default mode of brain function. Proc Natl Acad Sci U S A. (2001) 98:676-82. doi: 10.1073/pnas.98.2.676

54. Cao J, Chen X, Chen J, Ai M, Gan Y, Wang W, et al. Resting-state functional MRI of abnormal baseline brain activity in young depressed patients with and without suicidal behavior. J Affect Disord. (2016) 205:25263. doi: 10.1016/j.jad.2016.07.002

55. Hamilton JP, Farmer M, Fogelman P, Gotlib IH. Depressive rumination, the default-mode network, and the dark matter of clinical neuroscience. Biol Psychiatry. (2015) 78:224-30. doi: 10.1016/j.biopsych.2015.02.020

56. Duman RS, Aghajanian GK, Sanacora G, Krystal JH. Synaptic plasticity and depression: new insights from stress and rapid-acting antidepressants. Nat Med. (2016) 22:238-49. doi: 10.1038/nm.4050

57. Mason MF, Norton MI, Van Horn JD, Wegner DM, Grafton ST, Macrae CN. Wandering minds: the default network and stimulus-independent thought. Science. (2007) 315:393-5. doi: 10.1126/science.1131295

58. Ochsner KN, Ray RR, Hughes B, McRae K, Cooper JC, Weber J, et al. Bottom-up and top-down processes in emotion generation: common and distinct neural mechanisms. Psychol Sci. (2009) 20:1322-31. doi: 10.1111/j.1467-9280.2009.02459.x

59. Tymofiyeva O, Connolly CG, Ho TC, Sacchet MD, Henje Blom E, LeWinn KZ, et al. DTI-based connectome analysis of adolescents with major depressive disorder reveals hypoconnectivity of the right caudate. J Affect Disord. (2016) 207:18-25. doi: 10.1016/j.jad.2016.09.013
60. Khan AR, Chuhutin A, Wiborg O, Kroenke CD, Nyengaard JR, Hansen B, et al. Biophysical modeling of high field diffusion MRI demonstrates microstructural aberration in chronic mild stress rat brain. Neuroimage. (2016) 142:421-30. doi: 10.1016/j.neuroimage.2016.07.001

61. Cheng Y, Xu J, Arnone D, Nie B, Yu H, Jiang H, et al. Resting-state brain alteration after a single dose of SSRI administration predicts 8-week remission of patients with major depressive disorder. Psychol Med. (2017) 47:43850. doi: 10.1017/S0033291716002440

62. Guo W, Liu F, Zhang J, Zhang Z, Yu L, Liu J, et al. Abnormal default-mode network homogeneity in first-episode, drug-naive major depressive disorder. PLoS ONE. (2014) 9:e91102. doi: 10.1371/journal.pone.0091102

63. Yang W, Chen Q, Liu P, Cheng H, Cui Q, Wei D, et al. Abnormal brain activation during directed forgetting of negative memory in depressed patients. J Affect Disord. (2016) 190:880-8. doi: 10.1016/j.jad.2015.05.034

64. Wei X, Shen H, Ren J, Li X, Xu X, Yang R, et al. Altered resting-state connectivity in college students with nonclinical depressive symptoms. PLoS ONE. (2014) 9:e114603. doi: 10.1371/journal.pone.0114603

65. Chen G, Zhao L, Jia Y, Zhong S, Chen F, Luo X, et al. Abnormal cerebellumDMN regions connectivity in unmedicated bipolar II disorder. J Affect Disord. (2019) 243:441-7. doi: 10.1016/j.jad.2018.09.076

Conflict of Interest: The authors declare that the research was conducted in the absence of any commercial or financial relationships that could be construed as a potential conflict of interest.

Publisher's Note: All claims expressed in this article are solely those of the authors and do not necessarily represent those of their affiliated organizations, or those of the publisher, the editors and the reviewers. Any product that may be evaluated in this article, or claim that may be made by its manufacturer, is not guaranteed or endorsed by the publisher.

Copyright (C) $2022 \mathrm{Hou}, \mathrm{Li}, \mathrm{He}$, Zhang, Chen, Jiang, Yin and Yuan. This is an open-access article distributed under the terms of the Creative Commons Attribution License (CC BY). The use, distribution or reproduction in other forums is permitted, provided the original author(s) and the copyright owner(s) are credited and that the original publication in this journal is cited, in accordance with accepted academic practice. No use, distribution or reproduction is permitted which does not comply with these terms. 